

\title{
IoT Based Industrial Information Gathering
}

\author{
K. Bhuvaneswari \\ Lecturer Senior Grade, Department of Electrical and Electronics Engineering, \\ Nachimuthu Polytechnic College, Pollachi, Tamil Nadu, India
}

\begin{abstract}
This project aims to gather information from an industry by means of the internet of things (IOT). Internet of Things (IOT) is rapidly increasing technology. IOT is the network of physical objects or things embedded with electronics, software, sensors, and network connectivity, which enables these objects to collect and exchange data. In this project, We are developing a system which will automatically monitor the industrial application such as, Motor's speed, energy consuming of the industry, single phasing effect and no of loads connected, etc.,
\end{abstract}

This information's are uploaded on the internet user can view the information on the internet from anywhere and at any time. The data are stored in the internet cloud user can view it at any time. If the user found any single phasing in supply or some other issues user can get information from anywhere by means of internet. In this modern industries this technology

\section{Keywords: Ardunio, Internet, Sensors, Wi-Fi}

\section{INTRODUCTION}

Technological developments have enabled to be taken classic systems place by Automatic and advanced systems. In addition, the availability of fastprocessing, stable and sensitive products provided particular benefits in industrial automation. As a result of the developments in Communication technologies, systems are no longer monitored and controlled by personnel using classic methods, but automatically by computer-controlled or remote-controlled devices. Industrial environmental conditions have been upgrading day by day with this newly introduced automatic techniques as a result of getting rid of the conventional procedures of manufacturing increasing huge workloads. The next generation industries will be definitely more advanced and automatic as compared with existing ones.

This brings on a new terminology of "Smart Industries" in this new era of Monitoring as well as controlling of various Industrial applications. . As an emerging technology brought about rapid advances in modern wireless telecommunication, Internet of Things (IOT) has attracted a lot of attention and is expected to bring benefits to numerous applications. The newly introduced concept of "Internet of Things" (IOT) is providing a helping hand to achieve the Industrial automation through remote access. In IOT each device or devices constituting a system will be able to communicate with the other devices or system in the same premises over a common platform. Hence this leads to exchange of relevant data, statistics, logs and various other parameters information among various devices to improve their performance, which will help industries to have better productivity, management and increased throughput.

\subsection{BLOCK DIAGRAM}

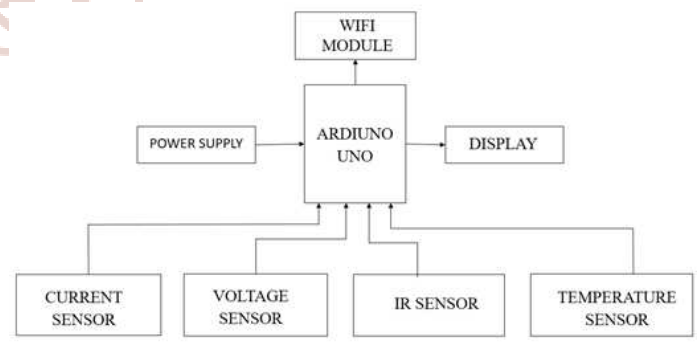

Figure 1: Block Diagram

\subsubsection{BLOCK DIAGRAM OPERATION Power Supply}

SMPS power supply adapter is used to provide power for the Arduino Uno. 


\section{Current Sensor}

The current sensor is interfaced with the Arduino Uno and. It is input terminals are connected in series with the main supply. By this the current is measured by the Arduino Uno.

\section{Voltage Sensor}

The voltage divider network is used as the voltage sensor. To measure the voltage in the circuit and the output of the network is interfaced with the Arduino Uno.

\section{IR Sensor}

The IR sensor is used for the measurement of speed of the motor. And its output interfaced with the microcontroller for count the number of pulse per minute. To calculate the speed in rotation per minute.

\section{Arduino UNO}

Arduino is an open-source electronics platform based on easy-to-use hardware and software. Arduino boards are able to read inputs and turn it into an output you can tell your board what to do by sending a set of instructions to the microcontroller on the board. To do so you use the Arduino programming language (based on Wiring), and the Arduino Software (IDE), based on Processing.

\section{Display}

Liquid crystal display is interfaced to microcontroller. Most commonly LCD used are $16^{*} 2 \& 20 * 2$ display means 16 represents column $\& 2$ represents rows.

\section{Wi-Fi Module}

ESP8266 Wi-Fi module is low cost standalone wireless transceiver that can be used for end-point IOT developments. ESP8266 Wi-Fi module enables internet connectivity to embedded applications. It uses TCP/UDP communication protocol to connect with server/client. To communicate with the ESP8266 WiFi module, microcontroller needs to use set of AT commands. Microcontroller communicates with ESP8266 Wi-Fi module using UART having specified Baud rate (Default 115200).

\section{Description}

In this project we upload the data from the sensors to cloud using Wi-Fi module. The project contains the block of sensors, microcontroller (Arduino Uno) and Wi-Fi module. The sensors. Are used to convert mechanical and electrical quantities into measurable signal. The microcontroller is used to convert the measurable signal into the real values of quantity and to produce output signal to upload the data in the cloud. The Wi-Fi module is used to upload the data to the cloud.

In this project have three modules they are

$>$ Input module

$>$ Processing module

$>$ Uploading module

It is used to explain the operation

\subsubsection{BLOCK DIAGRAM EXPLANATION Input module}

The input modules in this project are current sensor, voltage sensor, temperature sensor, ir sensor. These sensors are interfaced with the microcontroller sends an input signal to the microcontroller an input to the LCD display and to the Wi-Fi module.

\section{Processing Module}

The processing module in this project is microcontroller. The microcontroller used in this project is a developer board Arduino Uno R3. It converts the sensor values into the real values. And a signal is produced according to the real values calculated for serial transmission of data. This is for send to the Wi-Fi module for uploading process and LCD display.

\section{Uploading Module}

The uploading module is a Wi-Fi module. The Wi-Fi module used in this project is (esp8266). Which uploads the data to the cloud by means of internet connection, and the same time the LCD displays the data which is measured at real time.

\subsection{CIRCUIT DIAGRAM}

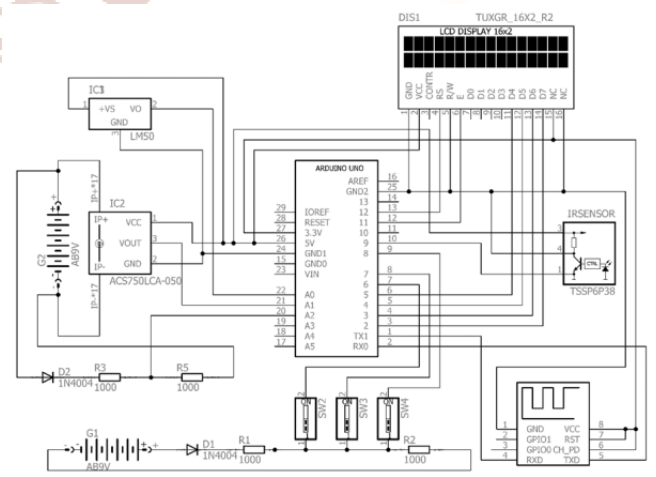

Figure 2: Circuit Diagram

\subsubsection{CIRCUIT DIAGRAM OPERATION}

The circuit has SMPS power supply. It provides the necessary supply for the microcontroller. And the 
current sensor voltage sensors IR sensors are provided by +5 volts and grounded on the microcontroller +5 volt pin and ground pin. The analogue data from the current sensor temperature sensor and voltage sensors are provided to the analogue pin in the Arduino Uno and the digital data from the IR sensor is provided to the digital pins in the Arduino Uno. The Wi-Fi module is interfaced with the microcontroller and it also operated at +5 voltages. The LCD display is interfaced with the microcontroller and the digital data is provided to display the measurement.

The temperature sensor (LM35) is interfaced with the Arduino Uno by means of an analogue pin (A0). It measures the temperature of the surface where it is placed. The sensor is provided by 5 volt form the Arduino Uno.

The current sensor (ASC721) is interfaced with the microcontroller Arduino Uno. By analogue pin (A1). It measures the current in the circuit. The ACS712 current sensor operates in the 5 volt which is provided by the $5 \mathrm{v}$ pin in the Arduino Uno.

The voltage divider network acts as a voltage sensor the Arduino Uno can measure voltage only up to 5 volts without sensor. By means of programmatic calculation of voltage divider network resistor range we can measure voltage up to 55 volts in Arduino Uno.

The IR sensor is used to measure speed of the motor. The digital data is generated in IR sensor module. The digital pin (10) is connected to OUT of the IR sensor module and number of pulses per minuets is calculated by programmatic calculation in the microcontroller. This module operates in 5 volts.

The Wi-Fi module is interfaced with the Arduino Uno. The TX and RX pins form the module is interfaced with the Arduino Uno TX and RX pins in order to transmit the serial data to the Wi-Fi module.

\subsection{SMPS POWER SUPPLY}

If we need DC power supply for circuits we choose step down transformer based Rectifier circuit, It may give constant DC voltage under Regulator ICs but when the current fluctuations occurs at Input Power Source then the DC output supply will also get affected. To get rid of this drawback in traditional DC power supply method engineers, electronic designers are go with SMPS circuit. You may heard the name
SMPS (Switched Mode Power Supply), It gives good constant DC output with considerably constant output current. This block diagram represents typical SMPS inner blocks.

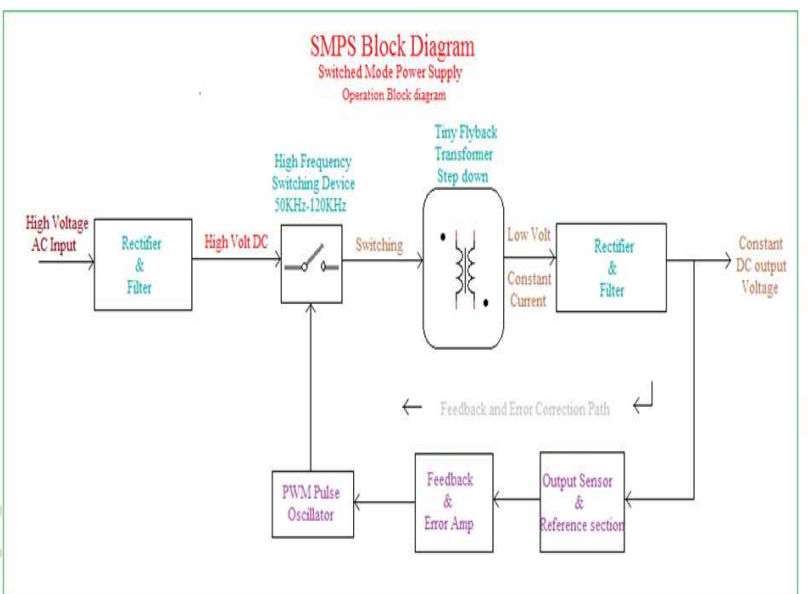

Figure 3: Block Diagram of SMPS

\subsection{ARDUINO UNO}

Arduino is an open-source electronics platform based on easy-to-use hardware and software. Arduino boards are able to read inputs - light on a sensor, a finger on a button, or a Twitter message - and turn it into an output - activating a motor, turning on an LED, publishing something online. You can tell your board what to do by sending a set of instructions to the microcontroller on the board. To do so you use the Arduino programming language (based on Wiring), and the Arduino Software (IDE), based on Processing. The Arduino board started changing to adapt to new needs and challenges, differentiating its offer from simple 8-bit boards to products for IOT applications. All Arduino boards are completely open-source, empowering users to build them independently and eventually adapt them to their particular needs. The software, too, is open-source, and it is growing through the contributions of users worldwide. The Arduino Uno is a microcontroller board based on the ATmega328p. It has 14 digital input/output pins (of which 6 can be used as PWM outputs), 6 analogue inputs, a $16 \mathrm{MHz}$ ceramic resonator, a USB connection, a power jack, an ICSP header, and a reset button. It contains everything needed to support the microcontroller. We either need to connect it to a computer using a USB cable or power it with an ACto-DC adapter. The Arduino circuit acts as an interface between the software part and the hardware part of the project.

In our project we programmed the Arduino Uno R3 to collect the data from the sensor and to convert it into real measured value as per the sensor output 
calculations. And it is programmed to display the real time information i.e. what process is currently occurring. In our project the Arduino Uno is also programmed to send the collected data to upload them to the Wi-Fi module ESP8266.

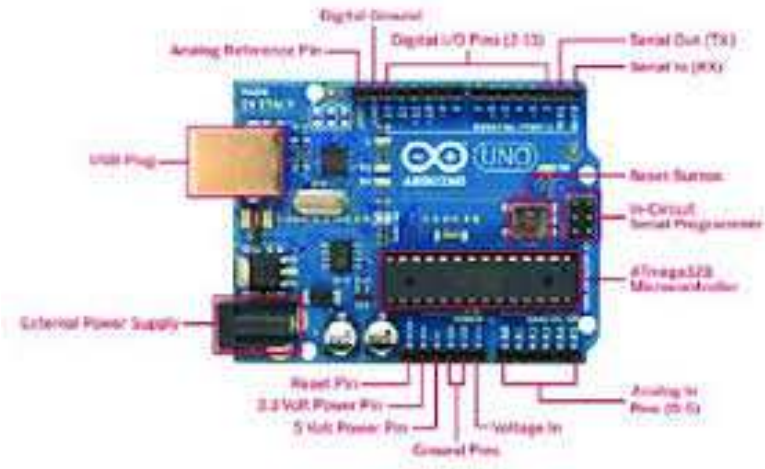

Figure 4: Pictorial View of Arduino Uno R3

\subsection{VOLTAGE DIVIDER NETWORK}

A voltage divider involves applying a voltage source across a series of two resistors. You may see it drawn a few different ways, but they should always essentially be the same circuit.



Figure 5: Circuit of Voltage Divider Schematics

\subsection{CURRENT SENSOR (ASC712)}

Typical applications include motor control, load detection and management, switch mode power supplies, and overcurrent fault protection. The device is not intended for automotive applications. The device consists of a precise, low-offset, linear Hall circuit with a copper conduction path located near the surface of the die. Applied current flowing through this copper conduction path generates a magnetic field which the Hall IC converts into a proportional voltage. Device accuracy is optimized through the close proximity of the magnetic signal to the Hall transducer. A precise, proportional voltage is provided by the low-offset, chopper-stabilized BiCMOS Hall IC, which is programmed for accuracy after packaging. The output of the device has a positive slope (>VI OUT (Q)) when an increasing current flows through the primary copper conduction path (from pins 1 and 2, to pins 3 and 4), which is the path used for current sampling. The internal resistance of this conductive path is $1.2 \mathrm{~m} \Omega$ typical, providing low power losses. The thickness of the copper conductor allows survival of the device at up to $5 \times$ overcurrent conditions. The terminals of the conductive path are electrically isolated from the signal leads (pins 5 through 8). This allows the ACS712 to be used in applications requiring electrical isolation without the use of opto-isolators or other costly isolation techniques.

\section{techniques.}

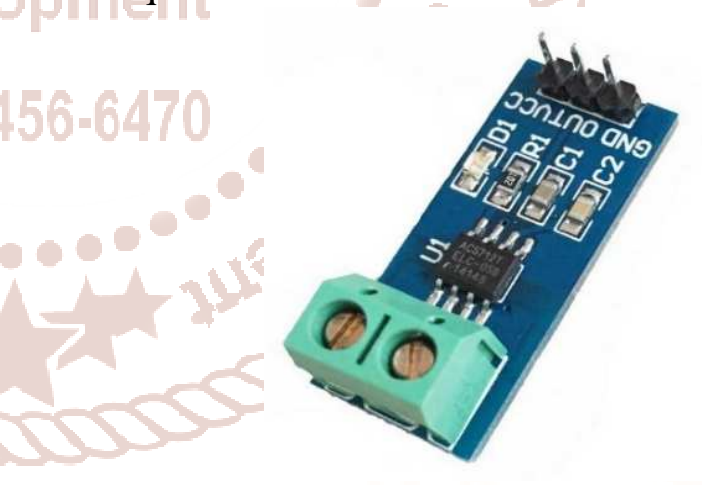

\section{Application of voltage divider network in this project}

In our project we are using voltage divider network for the measurement of voltage in the circuit because the analogue port in the Arduino Uno is designed to handle only 5 volts hence by using the voltage divider network we can measure up to 55 volts in the microcontroller. The voltage is measured in each phase and monitored this will help to manage the three phase supply to maintain the 3 phase supply in balanced load. Thus the single phase fault can be easily identified by measuring the phase voltage. The measured voltage is also used to measure the power.
Figure 6: Current Sensor Module ASC712

\subsection{IR SENSOR}

Indirect Incidence, both the IR LED and Photo diode are placed in parallel (side by side), facing both in same direction. In that fashion, when an object is kept in front of IR pair, the IR light gets reflected by the object and gets absorbed by photodiode.

In our project we using IR sensor module to measure the speed. Here the IR sensor is placed in front of the rotating wheel which is attached to the motor or drive where the speed to be measured. 


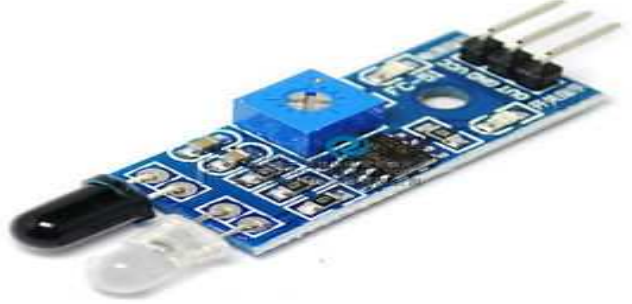

Figure 7: IR Sensor

\subsection{TEMPERATURE SENSOR}

LM35 temperature sensor has three terminals and required Maximum of $5.5 \mathrm{~V}$ supply. This type of sensor consists of a material that performs the operation according to temperature to vary the resistance. This change of resistance is sensed by circuit and it calculates temperature. When the voltage increases then the temperature also rises. We can see this operation by using a diode. Temperature sensors directly connected to microprocessor input and thus capable of direct and reliable communication with microprocessors. The sensor unit can communicate effectively with low-cost processors without the need of $\mathrm{A} / \mathrm{D}$ converters.

The LM35 series are precision integrated-circuit temperature sensors, whose output voltage is linearly proportional to the Celsius temperature. The LM35 is operates at $-55^{\circ}$ to $+120^{\circ} \mathrm{C}$. The basic centigrade temperature sensor $\left(+2^{\circ} \mathrm{C}\right.$ to shown in figure below

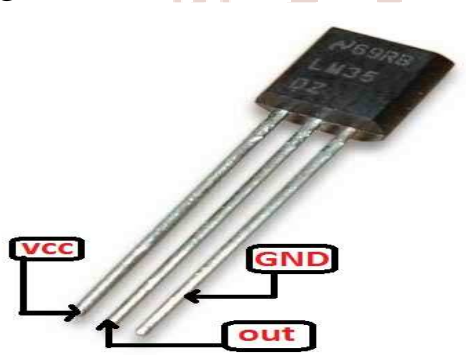

Figure 8: Temperature Sensor
$>$ Thus it measures the temperature of the motor and updates it to the microcontroller.

\subsection{WIFI MODULE ESP8266}

The ESP8266 Arduino compatible module is a lowcost Wi-Fi chip with full TCP/IP capability, and the amazing thing is that this little board has a MCU (Micro Controller Unit) integrated which gives the possibility to control $\mathrm{I} / \mathrm{O}$ digital pins via simple and almost pseudo-code like programming language. This little module allows the MCU to connect to $\mathrm{Wi}-\mathrm{Fi}$ network and create simple TCP/IP connections. The incredible small size attracted many geeks and hackers to explore it and use it in a large variety of projects. You can find over the internet hundreds of projects and various implementations like home automation, data logging solutions, robotics, controlling things over the internet, even drones or copters.

ESP8266 Technical specifications

- 32-bit RISC CPU: TensilicaXtensa LX106 running at $80 \mathrm{MHz} * *$

$64 \mathrm{KiB}$ of instruction RAM, $96 \mathrm{KiB}$ of data RAM

> External QSPI flash - $512 \mathrm{KiB}$ to $4 \mathrm{MiB}^{*}$ (up to $16 \mathrm{MiB}$ is supported)

IEEE $802.11 \mathrm{~b} / \mathrm{g} / \mathrm{n} \mathrm{Wi}$-Fi

Integrated TR switch, balun, LNA, power amplifier and matching network

- WEP or WPA/WPA2 authentication, or open networks

16 GPIO pins **

$>\mathrm{SPI}, \mathrm{I}^{2} \mathrm{C}$,

$>\mathrm{I}^{2} \mathrm{~S}$ interfaces with DMA (sharing pins with GPIO)

$>$ UART on dedicated pins, plus a transmit-only UART can be enabled on GPIO2

$>1$ 10-bit ADC

\section{Function of esp8266 in this project}

Features of LM35 Temperature Sensor:

$>$ Calibrated directly in ${ }^{\circ}$ Celsius (Centigrade)

$>$ Rated for full $1-55^{\circ}$ to $+150^{\circ} \mathrm{C}$ range

$>$ Suitable for remote applications

$>$ Low cost due to wafer-level trimming

$>$ Operates from 4 to 30 volts

$>$ Low self-heating,

$> \pm 1 / 4^{\circ} \mathrm{C}$ of typical nonlinearity

\section{Operation of LM35 in this project:}

$>$ The monitoring of the temperature in the industry is most important thing because the motor may get over heated which will cause the heavy economical and productivity unbalance.
The Wi-Fi module esp8266 is interfaced with the microcontroller to host the data to the internet. The website things speak is used to upload the data in this project. It is free website which provides an API key to host the data. The Wi-Fi module is connected to a common Wi-Fi which is declared during coding.



Figure 9: Wi-Fi Module 


\subsection{LIQUID CRYSTAL DISPLAY}

The $16 \times 2$ LCD display is a very basic module commonly used in DIYs and circuits. The $16 \times 2$ translates a display 16 characters per line in 2 such lines. In this LCD each character is displayed in a $5 \times 7$ pixel matrix.

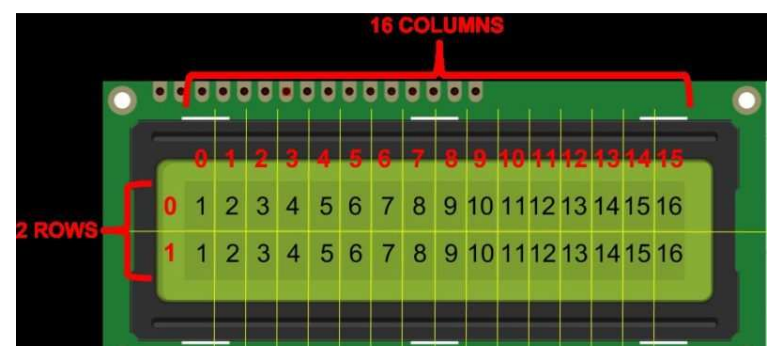

Figure 10: Liquid Crystal Display

The LCD display is used to display the current status to the sensor data and to display what process is occurring in the microcontroller and in the esp 8266. The microcontroller sends the digital data related to its function and input sensor data.

\subsection{ADVANTAGES}

$>$ Information's can be seen at any anywhere by means of internet.

$>$ Number of data can be increased by increasing the number of sensors in industry.

$>$ Easy for analysis of previous data.

$>$ In things speak website we can do Mat Lab analysis using uploaded data. Hence it will help slight increase in the productivity.

\subsection{APPLICATION}

$>$ Can be implemented in modern industry.

$>$ It can also implement instead of SCADA in the electrical grid system.

\section{CONCLUSION}

This project work is attempted to develop the monitoring system in the industrial sector. In this project IIOT industrial internet of things method is used. The industrial data's are collected by means of sensors and interfaced with the microcontroller Arduino Uno R3 and uploaded to the internet by means of Wi-Fi module ESP8266. In this project "things speak.com" is used for hosting and view the data.

In future the project idea can be implemented in all industries. By this the productivity and the current growth of the industries can be analysis with the previous data's.
The main feature scope of this project is to implement this sensor data collecting from all industries, electrical grids, residential and shops, etc. and upload them to cloud and they are analyzed for any current fault indication and also for the feature analysis in order to improve the productivity and also for avoid the wastage of power and product.

\section{REFERENCES}

1. Luigi A, Antonio I, Giacomo M. The Internet of Things: A survey. Elsevier Computer Networks. 2010 Jun; 54(15):2787-97.

2. Andrew $\mathrm{T}$, David W. Introduction. Computer Networks. PearsonEdu. Inc.: USA; 2010. p. 1-3.

3. Andrew T, David W. Uses of computer networks. Computer Networks. Pearson Edu. Inc.: USA. 2010. p. 3-16.

4. History of technology [Internet]. Available from:

5. Jayavardhana G, Rajkumar B, Slaven M, MarimuthuP. Internet of Things (IOT): A vision, architectural elements, and future directions. Elsevier Future Generation Computer Systems. $2013 \mathrm{Feb} ; 29(7): 1645-51$.

6. Roy W. An Introduction to RFID Technology. IEEE Pervasive Computing. 2006; 5(1):25-9.

7. Badri N, Franklin R, Roy W. RFID Technology and Applications. IEEE Pervasive Computing. 2006; 5(1):22-3.

8. - That 'Internet of Things' Thing [Internet]. [Cited 2016 Apr04]. Available from: 4986.

9. Prakash M, Gowshika U, Ravichandran T. A smart device integrated with an android for alerting a person's health condition: Internet of Things. Indian Journal of Science and Technology. 2016 Feb; 9(6):1-2, 5.

10. Bluetooth [Internet]. Available from: https://www.bluetooth.com/what-is-bluetoothtechnology/bluetooth.

11. Tom K. Basic sensor technology. Sensor Technology Handbook. Newnes: USA; 2004. p. 1.

12. Jacob F. Sensors, signals, and systems. Handbook of Modern Sensors. Springer: USA; 2010. p. 1-7.

13. Jacob F. Coupling with object. Handbook of Modern Sensors. Springer: USA; 2010. p. 519-20.

14. John F. Sensor types and technologies. Sensor Technology Handbook. Newnes: USA; 2004. p. 531-5. 
International Journal of Trend in Scientific Research and Development (IJTSRD) ISSN: 2456-6470

15. Jacob F. Acoustic temperature sensor; 21. Young HL, Raj M. Overview: What is a piezoelectric temperature sensors. Handbook of Modern Sensors. Springer: USA; 2010. p. 564-6. biosensor. Sensor Technology Handbook. Newnes: USA; 2004. p. 161-3.

16. Jacob F. Concepts of pressure. Handbook of Modern Sensors. Springer: USA; 2010. p. 375-6.

17. Glenn H. Piezoresistive pressure sensing. Sensor. Technology Handbook. Newnes: USA; 2004. p. 411-14.

22. Young HL, Raj M. Applications of biosensors. Sensor Technology Handbook. Newnes: USA; 2004. p. 164-8.

23. Jacob F. Acoustic sensors. Handbook of Modern Sensors. Springer: USA; 2010. p. 431.

18. Jacob F. Position, displacement, and level. Handbook of Modern Sensors. Springer: USA; 2010. p. 279-80.

24. John C, Larson D. Common microphone types. Sensor Technology Handbook. Newnes: USA; 2004. p. 482.

19. Adolfo CM. Proximity sensors. Sensor 25. Jacob F. Handbook of modern sensors.4th edn. Technology Newnes: USA. 2004; p. 345-9. Springer: USA; 2010.

20. Adolfo CM. Types of position sensors. Sensor Technology Handbook. Newnes: USA; 2004. p. 322 .

26. Jon SW. Sensor technology handbook. Newnes: USA; 2004.

27. Tom K. Sensor performance characteristics definitions. Sensor Technology Handbook. Newnes: USA; 2004. p. 2-4. 